\title{
TP53 mutations predict for poor survival in ALK rearrangement lung adenocarcinoma patients treated with crizotinib
}

\author{
Wen-Xian Wang ${ }^{1 \#}$, Chun-Wei Xu ${ }^{2 \#}$, Yan-Ping Chen ${ }^{2}$, Wei Liu ${ }^{2}$, Li-Hua Zhong ${ }^{2}$, Fang-Fang Chen ${ }^{2}$, Wu \\ Zhuang $^{3}$, Yun-Jian Huang ${ }^{3}$, Zhang-Zhou Huang ${ }^{3}$, Rong-Rong Chen ${ }^{4}$, Yan-Fang Guan ${ }^{4}$, Xin Yi ${ }^{4}$, Tang- \\ Feng $\mathrm{Lv}^{5}$, Wei-Feng Zhu ${ }^{2}$, Jian-Ping Lu ${ }^{2}$, Xiao-Jiang Wang ${ }^{2}$, Yi Shi ${ }^{2}$, Xian-Dong Lin ${ }^{2}$, Gang Chen ${ }^{2}$, Yong \\ Song ${ }^{5}$
}

${ }^{1}$ Department of Chemotherapy, Zhejiang Cancer Hospital, Hangzhou 310022, China; ${ }^{2}$ Department of Pathology, ${ }^{3}$ Department of Medical Oncology, Fujian Provincial Cancer Hospital, Fujian Medical University Cancer Hospital, Fuzhou 350014, China; ${ }^{4}$ Geneplus-Beijing, Beijing 102200, China; ${ }^{5}$ Department of Respiratory Medicine, Jinling Hospital, Nanjing 210002, China

Contributions: (I) Conception and design: W Zhuang, G Chen, TF Lv, Y Song; (II) Administrative support: RR Chen, YF Guan, X Yi; (III) Provision of study materials or patients: WX Wang, CW Xu, YP Chen, W Liu, LH Zhong, FF Chen, YJ Huang, ZZ Huang; (IV) Collection and assembly of data: WX Wang, CW Xu; (V) Data analysis and interpretation: WF Zhu, JP Lu, XJ Wang, Y Shi, XD Lin; (VI) Manuscript writing: All authors; (VII) Final approval of manuscript: All authors.

\#These authors contributed equally to this work.

Correspondence to: Wu Zhuang, MD, PhD. Department of Medical Oncology, Fujian Provincial Cancer Hospital, Fujian Medical University, No 420, Fuma Road, Fuzhou 350014, China. Email: zhuangwu2008@126.com; Gang Chen, MD, PhD. Department of Pathology, Fujian Provincial Cancer Hospital, Fujian Medical University, No 420, Fuma Road, Fuzhou 350014, China. Email: naichengang71@163.com.

Background: Advanced non-small cell lung cancer (NSCLC) patients who harbor anaplastic lymphoma kinase $(A L K)$ rearrangement are sensitive to an ALK inhibitor (crizotinib), but not all $A L K$-positive patients benefit equally from crizotinib treatment. We analyze the impact of TP53 mutations on response to crizotinib in patients with $A L K$ rearrangement NSCLC.

Methods: Sixty-six $A L K$ rearrangement NSCLC patients receiving crizotinib were analyzed. 21 cases were detected successfully by the next generation sequencing validation FFPE before crizotinib. TP53 mutations were evaluated in 8 patients in relation to disease control rate (DCR), objective response rate (ORR), progression-free survival (PFS) and overall survival (OS).

Results: TP53 mutations were observed in 2 (25.00\%), 1 (12.50\%), 1 (12.50\%) and 4 (50.00\%) patients in exons 5, 6, 7 and 8, respectively. The majority of patients were male $(75.00 \%, 6 / 8)$, less than 65 years old $(62.50 \%, 5 / 8)$ and never smokers $(75.00 \%, 6 / 8)$. ORR and DCR for crizotinib in the entire case series were $61.90 \%$ and $71.43 \%$, respectively. Statistically significant difference was observed in terms of PFS and OS between TP53 gene wild group and mutation group patients ( $\mathrm{P}=0.038, \mathrm{P}=0.021$, respectively).

Conclusions: TP53 mutations reduce responsiveness to crizotinib and worsen prognosis in $A L K$ rearrangement NSCLC patients.

Keywords: Non-small cell lung cancer (NSCLC); anaplastic lymphoma kinase (ALK); TP53; crizotinib

Submitted Jan 17, 2018. Accepted for publication Apr 09, 2018.

doi: $10.21037 /$ jtd.2018.04.98

View this article at: http://dx.doi.org/10.21037/jtd.2018.04.98 


\section{Introduction}

Lung cancer is one of the most common cancers and the leading cause of cancer death in the world (1). In the past decades, advances in molecular analysis and the development of targeted therapies have changed the identification of oncogenic drivers, the treatment and the survival for lung cancer. Anaplastic lymphoma kinase $(A L K)$ rearrangement is one of the driver genes which detected in $3-7 \%$ of patients with non-small cell lung cancer (NSCLC) (2). For these patients, ALK tyrosine kinase inhibitors (TKIs) have been developed. Crizotinib is a first generation ALK-TKI that shows efficacy in the treatment of $A L K$ rearrangement NSCLC (3). According to clinical studies of crizotinib (PROFILE 1005, PROFILE 1007, PROFILE1014), the major populations were lung adenocarcinoma (ADC) and an objective response rate (ORR) to crizotinib is approximately $60 \%$ and its median progression-free survival (PFS) is nearly 10 months (4). However, it is known that many cases ultimately acquired resistance to crizotinib (5). In addition, about $30 \% A L K$-positive patients to the treatment of crizotinib demonstrate primary resistance which early progressive disease (PD) after the first month of treatment $(6,7)$.

The mechanisms of acquired resistance included secondary mutations or copy number gain $(\mathrm{CNG})$ in the ALK kinase domain and up regulation of bypass signaling pathways (8). However, to our knowledge, the mechanisms of primary resistance to ALK inhibitor for these patients remain elusive. And only some primary resistance mechanisms have been hypothesized, such as mutations of the EGFR pathway and BIM polymorphisms (9-11).

According to reports, the tumor suppressor of TP53 gene mutations occur in approximately $25-50 \%$ NSCLC patients $(12,13)$. Ma et al. (14) has demonstrated regardless of EGFR and KRAS mutation status, non-disruptive TP53 mutations are independent markers of shorter overall survival (OS) in patients with advanced NSCLC. There is preclinical evidence in the human NSCLC cell lines NCI-H1299 and A549 showed a relationship between TP53 gene mutations and responsiveness to TKIs (15). Canale et al. (16) had reported that TP53 mutations reduce responsiveness to EGFR-TKIs and poor prognosis in EGFR-mutated NSCLC patients.

However, the relationship of TP53 gene status and the efficacy of crizotinib in $A L K$ positive NSCLC patients was unclear. In this study we proposed to evaluate the role of TP53 mutation in ALK rearrangement advanced NSCLC patients that received crizotinib treatment. We analyzed the status of TP53 gene and its association with the effect of crizotinib in Chinese patients with $A L K$-positive NSCLC.

\section{Methods}

\section{Patients}

Eligible patients were required to have pathologically confirmed NSCLC and sufficient tissue for analysis. Clinical and pathologic data prospectively collected for analyses included age at diagnosis, gender, smoking status, and stage according to the new International Association for the Study of Lung Cancer/American Thoracic Society/European Respiratory Society multidisciplinary classification. This study was approved by the ethics committee of Fujian Provincial Cancer Hospital, Fujian Medical University Cancer Hospital, Fuzhou Fujian, China, and a written informed consent was obtained from each participant before the initiation of any study-related procedure.

\section{Targeted next-generation sequencing}

A total of 66 ALK positive patients treated with crizotinib. However, 28 specimens were not enough and 38 specimens were evaluated by NGS. For 38 patients, including parts of patients with $A L K$ positive pts treatment before crizotinib, targeted region capture combined NGS was successfully performed in 21 patients. Genomic DNA sequencing libraries were prepared using the protocols recommended by the Illumina TruSeq DNA Library Preparation Kit. For samples close to the minimum input requirement, additional pre-capture PCR cycles were performed to generate sufficient PCR product for hybridization. The libraries were hybridized to custom-designed probes (Integrated DNA Technology) including all exons of 170 genes and selected intron of ALK, RET and ROS1 for the detection of Genomic rearrangements. DNA sequencing was performed on a HiSeq3000 sequencing system (Illumina, San Diego, CA) with $2 \times 75$ bp pairedend reads. The reads were aligned to the human genome build GRCh37 using BWA (a Burrows-Wheeler aligner). Somatic single nucleotide variant (sSNV) and indel calls were generated using MuTect and GATK, respectively. Somatic copy number alterations were identified with CONTRA. Genomic rearrangements were identified by the software developed in house analyzing chimeric read pairs. 


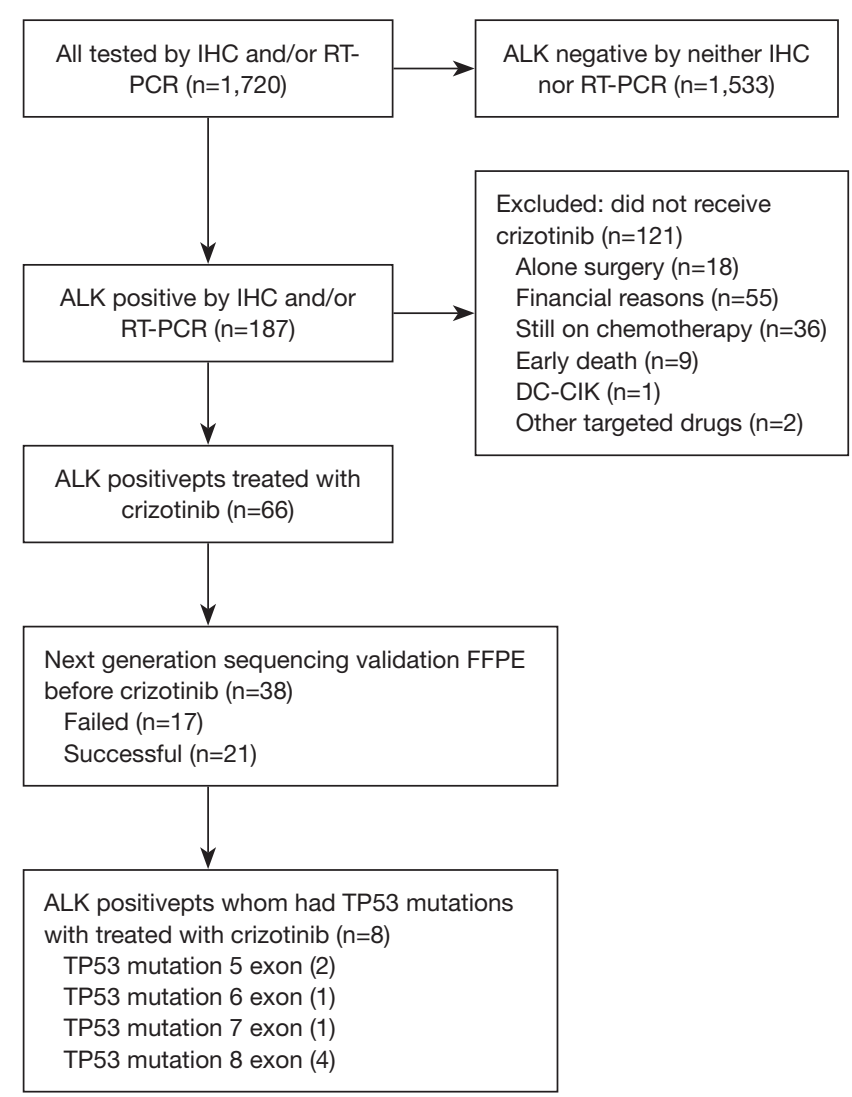

Figure 1 Flow chart of the study design.

\section{Response evaluation}

Patients received crizotinib treatment $(250 \mathrm{mg}$, twice daily) and had clinical data available including general characteristics, treatment efficacy and adverse reactions to treatment. Imaging data were independently reviewed by authors to evaluate their treatment responses according to the Response Evaluation Criteria in Solid Tumors (RECIST) version 1.1 PFS was calculated from the date of initiating targeted drugs treatment to radiologic or clinical observation of disease progression.

\section{Statistical analyses}

The response rate among subgroups and survival was described with Kaplan-Meier methodology and the logrank test was used to compare survival among subgroups. Statistical analysis was performed using SPSS version 19.0 software (IBM, Armonk, NY, USA). All P values were 2 -sided, and a $\mathrm{P}$ value of $<0.05$ was considered statistically significant.

\section{Results \\ Patient characteristics}

From July 2013 to May 2016, a total of 1,720 patients were enrolled in this study. Among them, 187 (10.87\%) were identified as $A L K$-positive and 66 of them received oral crizotinib. Because of the tissue sample insufficient and specimen quality testing, 21 of them was successfully performed by targeted region capture combined NGS. The flow chart of the study design is shown in Figure 1. Clinic-pathological characteristics of patients were reported in Table 1 . The majority of patients were male $(52.38 \%$, $11 / 21)$, less than 65 years old $(61.90 \%, 13 / 21)$ and never smokers $(80.95 \%, 17 / 21)$. All patients had a diagnosis of ADC $(100 \%, 21 / 21)$. All patients received crizotinib (100\%, 21/21). NGS detection found many accompanied genes, including mTOR pathway (PIK3CA p.E545K, FBXW7 p.R505L, STK11 p.D194E, PTEN p.R130G, MTOR p.E1799K, MTOR p.P2273L), RAS pathway (NRAS p.G12D), TP53 exon 5 (p.R158L, p.H179R), exon 6 (p.R213Q), exon 7 (p.G245S), exon 8 (p.Y271*, p.V272M, p.R273H, p.C277F) (Figure 2), SMARCA4 (p.R451L, p.E882K, p.R469W), $A R$ (p.E355K), APC (p.P2559L, p.T1910S), CCND1 (p.M82V), CTNNB1 (p.S45P), BRAF (p.D594G), RB1 (p.G449E) and NF1 (p.A2437S).

\section{TP53 mutation}

Out of the 21 patients with successfully targeted region capture combined NGS, 8 (38.10\%) patients showed a TP53 mutation: $25.00 \%$ (2/8) were on exon $5,12.50 \%$ $(1 / 8)$ on exon $6,12.50 \%(1 / 8)$ on exon 7 , and $50.00 \%(4 / 8)$ on exon 8 (Table 1). The majority of patients were male $(75.00 \%, 6 / 8)$, less than 65 years old $(62.50 \%, 5 / 8)$ and never smokers $(75.00 \%, 6 / 8)$. There were no significant differences noted with regard to age, sex, smoking history, ECOG PS, histology, number of previous treatments, between patients with and without the TP53 gene (Table 2).

\section{TP53 mutation and response to crizotinib}

ORR and disease control rate (DCR) for crizotinib in the entire case series were $61.90 \%$ and $71.43 \%$, respectively. TP5 3 gene wild group was found to be significantly associated with a higher ORR and DCR to crizotinib with respect to TP53 gene mutation group. An ORR of $76.92 \%$ and a DCR of $84.62 \%$ were observed in patients with TP53 
Table 1 Follow-up survival of 21 patients with NSCLC with TP53 gene status received crizotinib by targeted next-generation sequencing

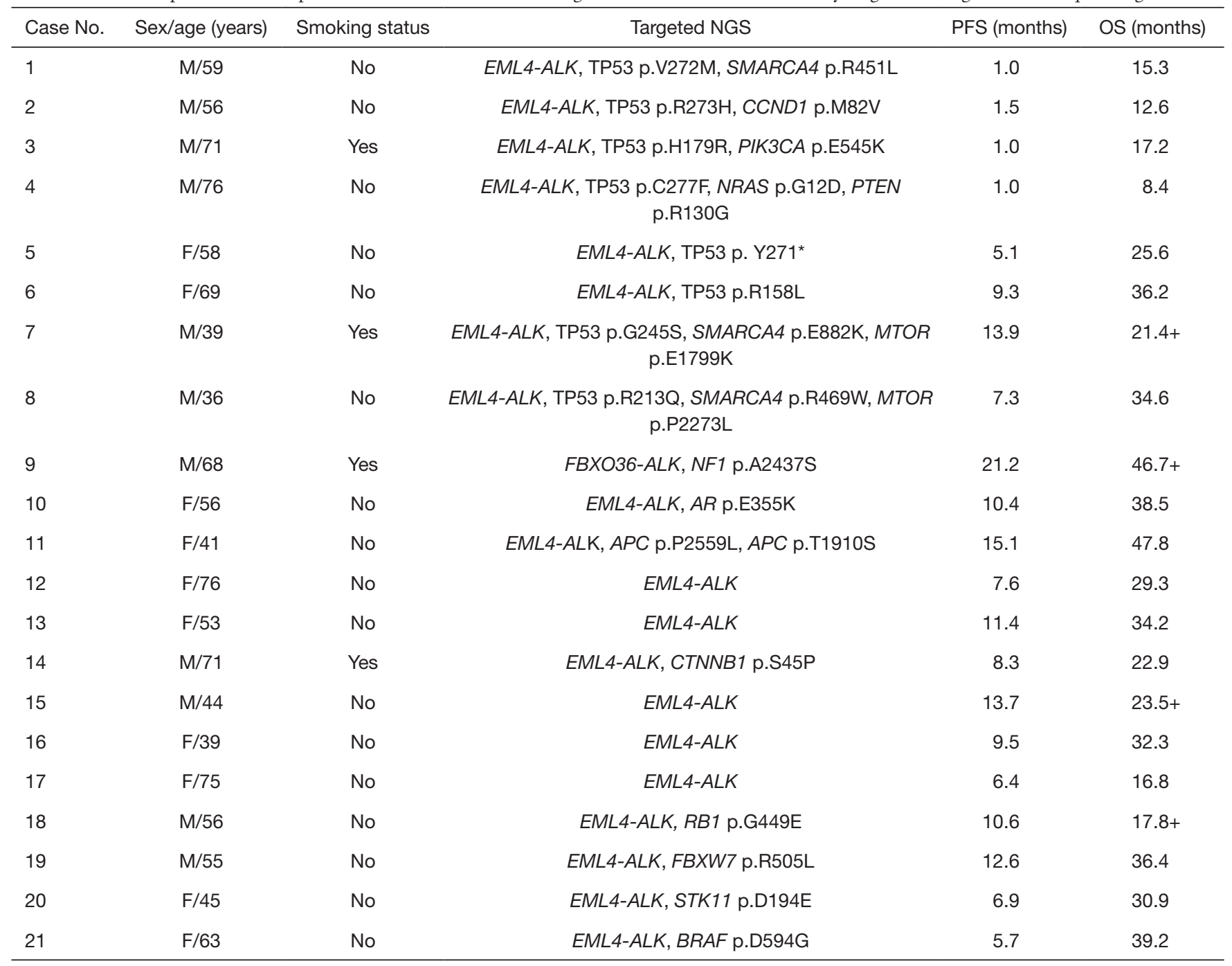

${ }^{*}$, representation oversight (NA). NSCLC, non-small lung cancer cell; M, male; F, female; NGS, next-generation sequencing; PFS, progressionfree survival; OS, overall survival.

gene wild group, and an ORR of $37.50 \%$ and a DCR of $50.00 \%$ were observed in patients with TP53 gene mutation group (Table 3).

\section{TP53 mutation and survival}

Statistically significant difference was observed in terms of PFS and OS between TP53 gene wild group and mutation group patients $(\mathrm{P}=0.038, \mathrm{P}=0.021$, respectively). The $\mathrm{PFS}$ in TP53 gene mutation group patients was shorter than that in TP53 gene wild group (3.3 vs. 10.4 months). The OS of TP53 gene mutation group was poorer than TP53 gene wild group (21.4 vs. 34.2 months). PFS and OS of TP53 gene on exon 5 were 9.3, 36.2 months and 1.0, 17.2 months), respectively; PFS and OS of TP53 gene on exon 6 were 7.3 and 34.6 months; PFS and OS of TP53 gene on exon 7 were 13.9 months, more than 21.4 months; PFS and OS of TP53 gene on exon 8 were 5.1 and 25.6 months, 1.0 and 15.3 months, 1.5 and 12.6 months, and 1.0 and 8.4 months, respectively (Table 1, Figure 3).

\section{Discussion}

To our knowledge, our study is the first to explore the relationship between TP53 gene mutation status and the effect of crizotinib in Chinese patients with $A L K$-positive advanced 

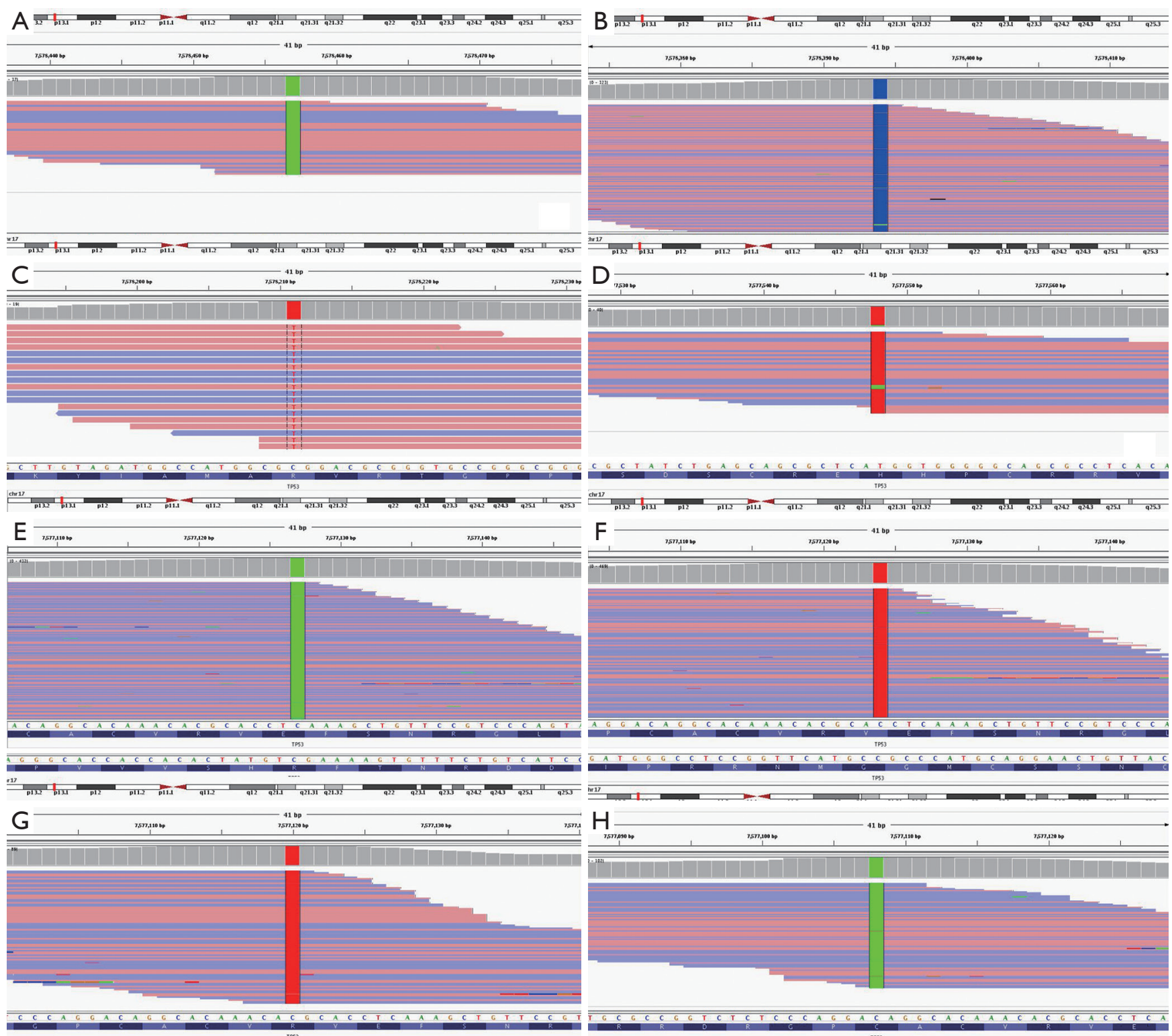

Figure 2 Targeted next-generation sequencing of TP53 gene IGV (A) exon 5 R158L, (B) exon 5 H179R, (C) exon 6 R213Q, (D) exon 7 G245S, (E) exon 8 E271*, (F) exon 8 V272M, (G) exon 8 R273H, (H) exon 8 C277F). *, representation oversight (NA).

NSCLC. NSCLC patients with $A L K$ rearrangement are highly sensitive to crizotinib. Nowadays, a series of trial data (phase I-III) involving in ALK positive advanced NSCLC patients demonstrated the efficacy of crizotinib was good and adverse reactions could be tolerated $(4,17,18)$. The data from East Asian patients also showed the ORR to crizotinib was $88 \%$ and its median PFS was 11.1 months (19). Unfortunately, some $A L K$-positive patients have been with shorter PFS after treatment with crizotinib which might be presence primary resistance to crizotinib. Up to now, the mechanisms of acquired resistance to ALK inhibitors can be divided into 2 types: ALK dominant or ALK non-dominant. ALK dominant includes secondary mutations and CNG in the $A L K$ gene; ALK non-dominant includes the activation of bypass tracks, such as EGFR, MET, KIT, KRAS and IGF$1 R(8)$. However, it is unclear that the causes of short PFS in ALK positive patients after crizotinib treatment. Some studies have explored the mechanisms of crizotinib primary resistance. Zhang et al. (9) reported patients with the BIM deletion polymorphism had a significantly shorter PFS (182 
vs. 377 days, $\mathrm{P}=0.008)$ and lower ORR (44.4\% vs. $81.7 \%$, $\mathrm{P}=0.041)$ compared with those without in 69 ALK/ROS1 positive patients with crizotinib treatment. Doebele et al. (10) reported that a patient received re-biopsy after 61 days on crizotinib which showed stable disease. This biopsy demonstrated a lack of an $A L K$ gene rearrangement by FISH, but the presence of an EGFR exon 21 mutation by direct

Table 2 The clinical characteristics of $A L K$ positive NSCLC with TPS3 gene status

\begin{tabular}{|c|c|c|c|}
\hline \multirow[b]{2}{*}{ Variable } & \multicolumn{2}{|c|}{ TP53 status } & \multirow[b]{2}{*}{$\mathrm{P}$} \\
\hline & $\begin{array}{l}\text { Positive } \\
\qquad(n=8)\end{array}$ & $\begin{array}{c}\text { Negative } \\
(n=13)\end{array}$ & \\
\hline Age, median [range], years & $58[36-76]$ & 57 [39-76] & 1.000 \\
\hline$<65$ & 5 & 9 & \\
\hline$\geq 65$ & 3 & 4 & \\
\hline Sex & & & 0.239 \\
\hline Male & 6 & 5 & \\
\hline Female & 2 & 8 & \\
\hline Smoking history & & & 0.802 \\
\hline Non-smoker & 7 & 13 & \\
\hline Smoker & 1 & 0 & \\
\hline ECOG PS & & & 0.688 \\
\hline $0-1$ & 8 & 11 & \\
\hline$\geq 2$ & 0 & 2 & \\
\hline Histology & & & 0.802 \\
\hline Adenocarcinoma & 7 & 13 & \\
\hline Non-adenocarcinoma & 1 & 0 & \\
\hline No. of previous treatments & & & 1.000 \\
\hline $0-1$ & 3 & 5 & \\
\hline$\geq 2$ & 5 & 8 & \\
\hline
\end{tabular}

ALK, anaplastic lymphoma kinase; NSCLC, non-small lung cancer cell; ECOG PS, Eastern Cooperative Oncology Group performance status. sequencing. There is still a lack in the mechanisms reports of primary resistance to crizotinib with $A L K$-positive NSCLC.

It is reported that TP53 gene mutations occur in approximately $50 \%$ of lung cancer patients and are more common in squamous cell lung carcinoma than in lung ADC (12). In the lung ADC cancer, TP53 mutation rates ranges from $25 \%$ to $40 \%(20,21)$. In addition, some reports showed the prevalence of ALK-rearranged with p53 mutation was about $9.1 \%(1 / 11)$ to $28.6 \%(2 / 7)(22,23)$. In our study, we observed a mutation percentage of $38.1 \%$ in $21 A L K$ positive ADC patients. Therefore, coexisting with TP53 mutations in ALK positive ADC patients is still further to explore in a larger research, especially for comparing ALK rearrangement patients with ALK negative patients. The $\mathrm{p} 53$ protein regulates cellular response to a variety of cellular stress signals by inducing cell cycle arrest (24). The normal function disruption of p53 disrupts this cellular response, leading to possible malignant cell transformation. Gene mutations lead to a loss of p53 functions, however non-disruptive mutations could remain some of the p53 protein functional properties (25). Therefore, we analyzed the relationship of efficacy and survival between $A L K$ positive and TP53 gene status with crizotinib therapy.

It is the first time to put forward that patients with TP53 gene mutation can no response to crizotinib. In our study, TP53 gene mutation group was found to be significantly associated with a lower ORR (76.92\% vs. 37.50\%) and DCR (50.00\% vs. $84.62 \%)$ to crizotinib with respect to TP53 gene wild group. In addition, significant shorter PFS (3.3 vs. 10.4 months, $\mathrm{P}=0.038)$ and $\mathrm{OS}(21.4$ vs. 34.2 months, $\mathrm{P}=0.021)$ were observed in patients with TP53 gene mutation group patients than TP53 gene mutation group. In particular, 4 of 8 TP53 mutated patients showed PD within two months of crizotinib therapy. Therefore, we think $A L K$ positive patients with TP53 gene mutation would influence the efficacy of crizotinib treatment. Likewise, in EGFR mutation populations, Canale et al. (16) also demonstrated TP53 mutations were associated with a significantly lower DCR

Table 3 Efficacy of crizotinib in patients with 21 cases of $A L K$ positive NSCLC positive patients with TP5 3 gene status

\begin{tabular}{lccccccc}
\hline TP53 gene status & CR & PR & SD & PD & ORR/\% & DCR/\% & PFS (months) \\
\hline TP53 wild & 0 & 10 & 1 & 2 & 76.92 & 84.62 & 10.4 \\
TP53 mutation & 0 & 3 & 1 & 4 & 37.50 & 50.00 & 3.3
\end{tabular}

ALK, anaplastic lymphoma kinase; NSCLC, non-small lung cancer cell; CR, complete response; PR, partial response; SD, stable disease; PD, progression disease; ORR, objective response rate; DCR, disease control rate; PFS, progression-free survival. 

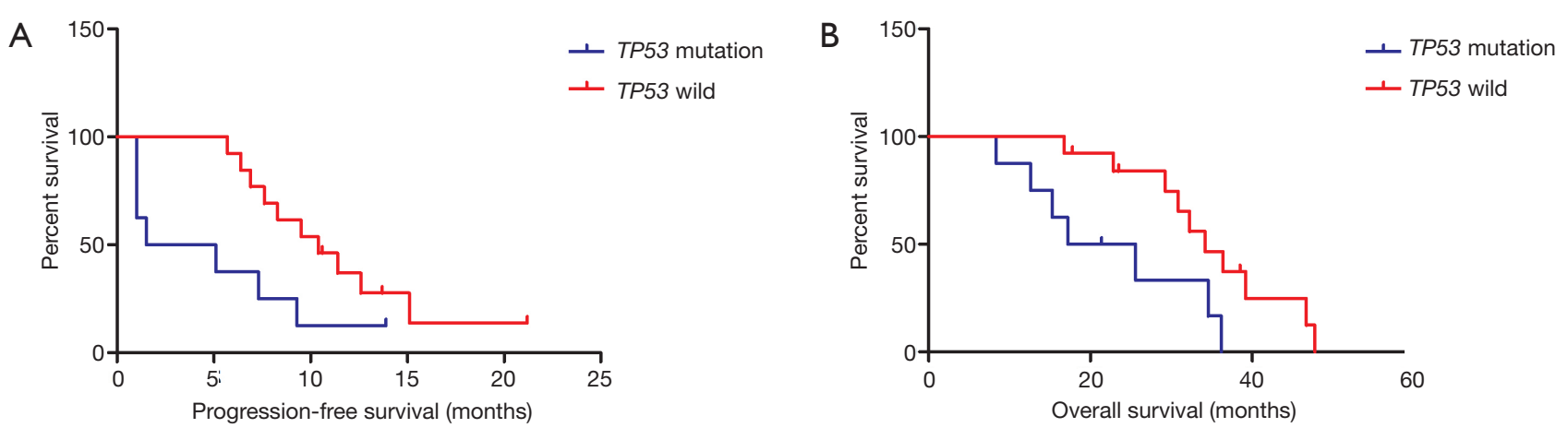

Figure 3 Progression-free survival (A) and overall survival (B) of crizotinib in anaplastic lymphoma kinase $(A L K)$ positive adenocarcinoma.

respect to wild patients. However, no statistically significant difference was observed in terms of PFS and OS between two groups. Their most significant result was that TP53 exon 8 mutation was associated with the worst prognosis. Our study also found $A L K$ positive patients with TP53 exon 8 mutation were poorer survival than other mutation types. The TP53 mutant type has been found to be an important factor for gefitinib-induced apoptosis in NSCLC cell lines and reduces gefitinib-induced apoptosis (26). We think that TP53 could have a role as prognostic factor to ALK inhibitors, rather than predictive factor. In the future, we should carry out well-designed prospective multicenter studies to demonstrate the correlation between TP53 gene status and ALK inhibitors including other generations in patients with $A L K$-positive disease.

We also recognize that there are several limitations to our study. First, due to a small sample, the conclusions should be bias a certain extent. Second, this was a retrospective study, and therefore selection bias was inevitable. Hence, future research will continue to increase the population in the hope of reducing the bias to some extent.

In conclusion, we described the relationship between TP53 gene and the efficacy of crizotinib in $A L K$ rearrangement patients. And the TP5 3 mutant patients were associated with poor ORR and shorter PFS. However, more work and further studies should be performed to explore the significance of TP53 gene in patients with $A L K$-positive NSCLC who were treated with ALK inhibitors.

\section{Acknowledgements}

Funding: This study was supported in part by grants from the National Clinical Key Specialty Construction Program (2013); Leading Project Foundation of Science Department of Fujian Province (2016Y0019); Leading Project
Foundation of Science Department of Fujian Province (2015Y0011).

\section{Footnote}

Conflicts of Interest: The authors have no conflicts of interest to declare.

Ethical Statement: This study was approved by the ethics committee of Fujian Provincial Cancer Hospital, Fujian Medical University Cancer Hospital, Fuzhou Fujian, China, and a written informed consent was obtained from each participant before the initiation of any study-related procedure.

\section{References}

1. Siegel RL, Miller KD, Jemal A. Cancer statistics, 2017. CA Cancer J Clin 2017;67:7-30.

2. Ettinger DS, Wood D, Akerley W, et al. Non-Small Cell Lung Cancer, Version 6. 2015. J Natl Compr Canc Netw 2015;13:515-24.

3. Sullivan I, Planchard D. Treatment modalities for advanced ALK-rearranged non-small-cell lung cancer. Future Oncol 2016;12:945-61.

4. Shaw AT, Kim DW, Nakagawa K, et al. Crizotinib versus chemotherapy in advanced ALK-positive lung cancer. $\mathrm{N}$ Engl J Med 2013;368:2385-94.

5. Toyokawa G, Seto T. Updated Evidence on the Mechanisms of Resistance to ALK Inhibitors and Strategies to Overcome Such Resistance: Clinical and Preclinical Data. Oncol Res Treat 2015;38:291-8.

6. Gainor JF, Dardaei L, Yoda S, et al. Molecular mechanisms of resistance to first- and second-generation ALK inhibitors in ALK-rearranged lung cancer. Cancer Discov 
2016;6:1118-33.

7. Steuer CE, Ramalingam SS. ALK-positive non-small cell lung cancer: mechanisms of resistance and emerging treatment options. Cancer 2014;120:2392-402.

8. Katayama R, Shaw AT, Khan TM, et al. Mechanisms of acquired crizotinib resistance in ALK-rearranged lung cancers. Sci Transl Med 2012;4:120ra17.

9. Zhang L, Jiang T, Li X, et al. Clinical features of Bim deletion polymorphism and its relation with crizotinib primary resistance in Chinese patients with ALK/ROS1 fusion-positive non-small cell lung cancer. Cancer 2017. [Epub ahead of print].

10. Doebele RC, Pilling A, Aisner D, et al. Mechanisms of resistance to crizotinib in patients with ALK gene rearranged non-small cell lung cancer. Clin Cancer Res 2012;18:1472-82.

11. Wang W, Jiang X, Song Z, et al. Patients harboring EGFR mutation after primary resistance to crizotinib and response to EGFR-tyrosine kinase inhibitor. Onco Targets Ther 2016;9:211-5.

12. Mogi A, Kuwano H. TP53 mutations in non small cell lung cancer. J Biomed Biotechnol 2011;2011:583929.

13. Deben C, Deschoolmeester V, Lardon F, et al. TP53 and MDM2 genetic alterations in non-small cell lung cancer: Evaluating their prognostic and predictive value. Crit Rev Oncol Hematol 2016;99:63-73.

14. Molina-Vila MA, Bertran-Alamillo J, Gasco A, et al. Nondisruptive p53 mutations are associated with shorter survival in patients with advanced non-small cell lung cancer. Clin Cancer Res. 2014; 20: 4647-59.

15. Rho JK, Choi YJ, Ryoo BY, et al. p53 enhances gefitinibinduced growth inhibition and apoptosis by regulation of fas in non-small cell lung cancer. Cancer Res 2007;67:1163-9.

16. Canale M, Petracci E, Delmonte A, et al. Impact of TP53 Mutations on Outcome in EGFR-Mutated Patients Treated with First-Line Tyrosine Kinase Inhibitors. Clin Cancer Res 2017;23:2195-202.

Cite this article as: Wang WX, Xu CW, Chen YP, Liu W, Zhong LH, Chen FF, Zhuang W, Huang YJ, Huang ZZ, Chen RR, Guan YF, Yi X, Lv TF, Zhu WF, Lu JP, Wang XJ, Shi Y, Lin XD, Chen G, Song Y. TP53 mutations predict for poor survival in $A L K$ rearrangement lung adenocarcinoma patients treated with crizotinib. J Thorac Dis 2018;10(5):2991-2998. doi: $10.21037 /$ jtd.2018.04.98
17. Kwak EL, Bang YJ, Camidge DR, et al. Anaplastic lymphoma kinase inhibition in non-small-cell lung cancer. N Engl J Med 2010;363:1693-703.

18. Solomon BJ, Mok T, Kim DW, et al. First-line crizotinib versus chemotherapy in ALK-positive lung cancer. $\mathrm{N}$ Engl J Med 2014;371:2167-77.

19. Lu S, Mok T, Lu Y, et al. Phase 3 study of first-line crizotinib vs pemetrexed cisplatin/carboplatin (PCC) in East Asian patients (pts) with ALK+ advanced nonsquamous non-small cell lung cancer (NSCLC). J Clin Oncol 2016;34:abstr 9058.

20. Ma X, Le Teuff G, Lacas B, et al. Prognostic and predictive effect of TP53 mutations in non-small cell lung cancer patients from adjuvant cisplatin-based therapy randomized trials: A LACE-bio pooled analysis. J Thorac Oncol 2016;11:850-61.

21. Halvorsen AR, Silwal-Pandit L, Meza-Zepeda L A, et al. TP53 mutation spectrum in smokers and never smoking lung cancer patients. Front Genet 2016;7:85.

22. Tuononen K, Kero M, Mäki-Nevala S, et al. ALK fusion and its association with other driver gene mutations in Finnish non-small cell lung cancer patients. Genes Chromosomes Cancer 2014;53:895-901.

23. Inamura K, Takeuchi K, Togashi Y, et al. EML4-ALK lung cancers are characterized by rare other mutations, a TTF1 cell lineage, an acinar histology, and young onset. Mod Pathol 2009;22:508-15.

24. Zilfou JT, Lowe SW. Tumor suppressive functions of $\mathrm{p} 53$. Cold Spring Harb Perspect Biol 2009;1:a001883.

25. Poeta ML, Manola J, Goldwasser MA, et al. TP53 mutations and survival in squamous-cell carcinoma of the head and neck. N Engl J Med 2007;357:2552-61.

26. Deben C, Van den Bossche J, Van Der Steen N, et al. Deep sequencing of the TP53 gene reveals a potential risk allele for non-small cell lung cancer and supports the negative prognostic value of TP53 variants. Tumour Biol 2017;39:1010428317694327. 